\title{
Characterizing the Role of the Primary Care Provider in Preventive Health Exams: NAMCS 201 1-2014
}

\author{
Aarti Rao, $B A^{7}$ and Minal S. Kale, MD, MPH \\ ${ }^{1}$ Icahn School of Medicine at Mount Sinai, New York, NY, USA; ${ }^{2}$ Division of General Internal Medicine, Icahn School of Medicine at Mount Sinai, New \\ York, NY, USA.
}

J Gen Intern Med 34(8):1385-7

DOI: $10.1007 / \mathrm{s} 11606-019-04916-9$

(c) Society of General Internal Medicine 2019

\section{INTRODUCTION}

The Affordable Care Act (ACA) shifted US health policy to emphasize disease prevention by mandating full coverage of approved preventive services. ${ }^{1}$ However, there is considerable debate regarding the continued utility of preventive health exams (PHEs) in the era of new forms of primary care. ${ }^{2}$ To inform this debate, we sought to characterize the care delivered at the PHE. We compare the services provided during PHEs by primary care providers (PCPs) vs. non-primary care providers, and also examine the services provided at PHEs compared with those provided at visits for chronic and acute conditions.

\section{METHODS}

We performed a cross-sectional analysis using data from the 2011-2014 National Ambulatory Medical Care Survey (NAMCS). NAMCS is a nationally representative survey of visits to office-based physicians. We defined a visit as a PHE when the patient's reason for visit was for a general examination, the physician's reason for visit was preventive care, and the physician's only coded diagnosis was a general examination. We identified visits for chronic and acute conditions if the physician's reason for visit was defined as such on the NAMCS survey. We defined PCPs as providers within family/general practice, internal medicine, or pediatrics. Non-PCPs belonged to other medical specialties or obstetrics and gynecology (ob/gyn). We examined all 23 diagnostic and screening services and 7 counseling services included in the NAMCS survey. We calculated the weighted proportion of visits in which a patient received a particular service and compared proportions using $\chi^{2}$ testing.

\section{RESULTS}

Of the estimated 3,055,782,135 visits by adults between 2011 and $2014,64,662,713$ were for a PHE $(2.1 \%$; 95\% CI, 1.92.3). The majority of PHEs were conducted in either solo or

Published online March 11, 2019 group private practices $(90.22 \%$; 95\% CI, 87.5-92.4). In our comparison of services in PHEs conducted by PCPs vs. nonPCPs (Table 1), PCPs provided more dietary counseling (31.3\% vs. $17.5 \% ; p<0.001)$, exercise counseling $(29.1 \%$ vs. $15.0 \% ; p<0.001)$, depression screening ( $8.4 \%$ vs. $4.2 \%$; $p=0.01$ ), and colonoscopy referrals (5.6\% vs. $2.8 \% ; p=$ $0.004)$. PCPs also provided more screening CBCs $(42.6 \%$ vs. $30.3 \% ; p<0.001)$ and EKGs $(15.7 \%$ vs. $9.0 \% ; p=0.01)$ than non-PCPs. In our comparison of services across visit type (Table 2), we observed significantly higher rates of the following tests during PHEs: CBCs $(11.8 \%$ in acute vs. $13.1 \%$ in chronic vs. $37.4 \%$ in PHE; $p<0.001)$, EKGs $(3.1 \%$ in acute vs. $3.2 \%$ in chronic vs. $12.9 \%$ in PHE; $p<0.001$ ), and urinalyses $(8.1 \%$ in acute vs. $6.4 \%$ in chronic vs. $25.6 \%$ in PHE; $p<0.001)$.

\section{DISCUSSION}

Our findings help characterize the strengths and weaknesses of PCPs during the PHE and provide a broader look into the delivery of services during ambulatory visits.

Our results indicate that PCPs provided higher rates of counseling, depression screening, and colonoscopy referrals than non-PCPs during the PHE. Similar trends have been previously observed and are likely due to the trust established by the continuity of the PCP-patient relationship, and the training that generalist physicians receive that emphasizes preventive intervention. ${ }^{3}$ Non-PCPs provided higher rates of mammograms and Pap smears, which is unsurprising given our classification of ob/gyns as non-PCPs.

Compared with non-PCPs, PCPs also provided more tests that the United States Preventive Services Task Force (USPSTF) recommends against during a PHE, such as screening CBCs and EKGs. Despite the lack of evidence for mass screening with such tests as a means of prevention, many PCPs believe that screening laboratory tests detect subclinical illness in asymptomatic patients. ${ }^{4} \mathrm{We}$ observed similarly high rates of CBCs, EKGs, and urinalyses during PHEs than during visits for acute and chronic conditions, which may be due to misaligned incentives in our fee-for-service healthcare model.

Our study has several potential limitations. It is possible that some of the non-recommended screening tests were indicated 
Table 1 Comparison of PHE Services by PCPs vs. Non-PCPs in 2011-2014 in Adults $\geq 18$

\begin{tabular}{|c|c|c|c|}
\hline & Non-PCP conducted visit & PCP conducted visit & $p$ value \\
\hline No. of visits (weighted) & $27,186,400$ & $37,476,313$ & \\
\hline \multicolumn{4}{|l|}{ Health education/counseling } \\
\hline Diet/nutrition & 17.5 & 31.3 & $<0.001$ \\
\hline Exercise & 15.0 & 29.1 & $<0.001$ \\
\hline Family planning/contraception & 3.3 & 1.3 & 0.03 \\
\hline Tobacco use cessation & 3.3 & 7.1 & 0.004 \\
\hline Weight reduction & 4.4 & 9.3 & 0.002 \\
\hline Injury prevention & $*$ & 6.0 & $*$ \\
\hline \multicolumn{4}{|l|}{ Imaging } \\
\hline Mammography & 11.9 & 7.8 & 0.02 \\
\hline X-ray & 3.0 & 4.2 & 0.23 \\
\hline Bone density & 3.0 & 3.5 & 0.55 \\
\hline \multicolumn{4}{|l|}{ Blood tests } \\
\hline $\mathrm{CBC}$ & 30.3 & 42.6 & $<0.001$ \\
\hline PSA & 6.2 & 13.0 & $<0.001$ \\
\hline Lipid profile & 34.0 & 46.1 & $<0.001$ \\
\hline HbAlc & 10.2 & 14.8 & 0.11 \\
\hline Glucose & 14.9 & 14.7 & 0.95 \\
\hline \multicolumn{4}{|l|}{ Tests and procedures } \\
\hline Chlamydia test & 2.6 & 1.7 & 0.26 \\
\hline Colonoscopy & 2.8 & 5.6 & 0.004 \\
\hline $\mathrm{EKG} / \mathrm{ECG}$ & 9.0 & 15.7 & 0.01 \\
\hline PAP test & 21.1 & 7.2 & $<0.001$ \\
\hline Urinalysis & 23.5 & 27.2 & 0.52 \\
\hline \multicolumn{4}{|l|}{ Screening } \\
\hline Depression screening & 4.2 & 8.4 & 0.01 \\
\hline
\end{tabular}

*Fewer than 30 records were available, making the estimates unreliable

to investigate issues that arose during the PHE, but were not documented in the chart. There is also the possibility of misclassification and underreporting of visit content on the NAMCS survey.
Hopefully, recent policy initiatives to improve primary care access through changed reimbursement strategies, such as the medical home model, will lead to stronger PCP-patient relationships and improved quality of care through counseling and

Table 2 Comparison of Services in PHEs, Visits for Acute Problems, and Visits for Chronic Care in 2011-2014 in Adults $\geq 18$

\begin{tabular}{|c|c|c|c|c|}
\hline & Acute & Chronic & PHE & $p$ value $^{\dagger}$ \\
\hline No. of visits (weighted) & $1,337,206,061$ & $1,094,361,868$ & $64,662,713$ & \\
\hline \multicolumn{5}{|l|}{ Health education/counseling } \\
\hline Diet/nutrition & 7.0 & 11.0 & 25.5 & $<0.001$ \\
\hline Exercise & 4.7 & 8.2 & 23.2 & $<0.001$ \\
\hline Family planning/contraception & 0.7 & 0.4 & 2.2 & $<0.001$ \\
\hline Stress management & 1.3 & 2.4 & 2.5 & 0.50 \\
\hline Tobacco use cessation & 2.0 & 2.4 & 5.5 & $<0.001$ \\
\hline Weight reduction & 2.4 & 4.0 & 7.2 & $<0.001$ \\
\hline Injury prevention & 2.4 & 1.0 & 4.3 & 0.01 \\
\hline \multicolumn{5}{|l|}{ Imaging } \\
\hline CT scan & 3.3 & 1.5 & $*$ & $*$ \\
\hline Echocardiogram & 1.4 & 1.2 & 0.9 & 0.22 \\
\hline Mammography & 0.9 & 1.0 & 9.5 & $<0.001$ \\
\hline MRI & 1.99 & 1.48 & $*$ & $*$ \\
\hline X-ray & 10.9 & 3.7 & 3.7 & $<0.001$ \\
\hline Bone density & 0.4 & 0.5 & 3.2 & $<0.001$ \\
\hline \multicolumn{5}{|l|}{ Blood tests } \\
\hline $\mathrm{CBC}$ & 11.8 & 13.1 & 37.4 & $<0.001$ \\
\hline PSA & 1.9 & 2.1 & 10.1 & $<0.001$ \\
\hline Lipid profile & 6.8 & 9.9 & 41.1 & $<0.001$ \\
\hline HbAlc & 4.6 & 6.4 & 12.9 & $<0.001$ \\
\hline Glucose & 5.0 & 5.5 & 14.8 & $<0.001$ \\
\hline \multicolumn{5}{|l|}{ Tests and procedures } \\
\hline Cardiac stress test & 0.9 & 0.9 & $*$ & $*$ \\
\hline Chlamydia test & 0.7 & 0.1 & 2.1 & $<0.001$ \\
\hline Colonoscopy & 2.0 & 1.0 & 4.4 & $<0.001$ \\
\hline $\mathrm{EKG} / \mathrm{ECG}$ & 3.1 & 3.2 & 12.9 & $<0.001$ \\
\hline PAP test & 1.03 & 0.35 & 13.1 & $<0.001$ \\
\hline Urinalysis & 8.1 & 6.4 & 25.6 & $<0.001$ \\
\hline \multicolumn{5}{|l|}{ Screening } \\
\hline Depression screening & 2.0 & 3.1 & 6.6 & $<0.001$ \\
\hline
\end{tabular}

tp values are comparing both acute and chronic visits to the PHE

*Fewer than 30 records were available, making the estimates unreliable 
recommended screening tests. ${ }^{5}$ However, these policy initiatives will not suffice and will only thrive with concurrent efforts to bridge the gap between data-driven recommendations by organizations such as the USPSTF and providers' attitudes towards preventive screening.

Corresponding Author: Aarti Rao, BA; Icahn School of Medicine at Mount Sinai, New York, NY, USA (e-mail: aarti.rao@icahn.mssm.edu).

\section{Compliance with Ethical Standards:}

Conflict of Interest: The authors declare that they do not have a conflict of interest.
Publisher's Note: Springer Nature remains neutral with regard to jurisdictional claims in published maps and institutional affiliations.

\section{REFERENCES}

1. The Patient Protection and Affordable Care Act, P.L. 111-148, 23 March 2010.

2. Mehrotra A, Prochazka A. Improving value in health care-against the annual physical. N Engl J Med. 2015;373(16):1485-7.

3. Starfield B, Shi L, Macinko J. Contribution of primary care to health systems and health. Milbank Q. 2005;83(3):457-502.

4. Prochazka AV, Lundahl K, Pearson W, Oboler SK, Anderson RJ. Support of evidence-based guidelines for the annual physical examination: a survey of primary care providers. Arch Intern Med. 2005; 165(12):1347-52.

5. Rittenhouse DR, Shortell SM. The patient-centered medical home: will it stand the test of health reform? JAMA. 2009;301(19):2038-40. 